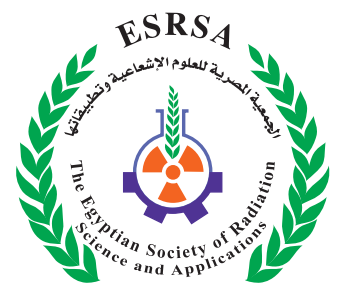

J. Nucl. Tech. Appl. Sci., Vol. 8, PP. $1: 6$ (2020)

\title{
Improving of Grid Interface with NPP Based on Optimal Capacitor Placement
}

\author{
Adail, A.S. ${ }^{1}$ and Nagy S.A. ${ }^{2}$
}

Received: $16 / 12 / 2019$

Accepted: 02/02/2020

E.mail:dr_adail@yahoo.com

\section{KEYWORDS}

NPP, Power Quality,

Reliability Grid,

Optimal Capacitor

Allocation.

\section{ABSTRACT}

The interface between Nuclear Power Plants (NPPs) and electric grids deserves careful attention, especially in low-performance electric power systems. NPP, during shutdown and startup, are in essential need to reliable electric energy that should be delivered by power grid to NPP power house. Safety systems of the NPP are designed for continuous operation with limited variations in voltage and frequency from the nominal values. Hence electrical supply voltage and frequency should also be controlled within a defined narrow range. One way to improve grid characteristics is using Capacitors devices which are placed in distribution networks with various sizes to improve voltage profile and to control reactive power. This paper proposes a method for optimization of capacitors location and sizes, to improve the voltage profile as well as to reduce power losses of electric grid connecting with NPP. The proposed technique is based on genetic algorithms and applied to IEEE16-bus radial distribution network to assure its improvement. The tabulated results show high efficiency in

1. Nuclear Fuel Technology Department, HotLab. Center, Atomic Energy Authority, Inshas, Egypt.

2. Electrical Engineering Department, University of Bisha, Saudi Arabia. 
electrical network (power loss reduction and voltage profile).

\section{INTRODUCTION}

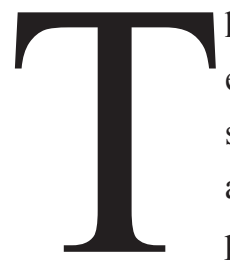

he electrical power systems are needed during all modes of operation: startup; normal operation; during and after reactor shutdown; and as a high priority source of power during certain nuclear events. Special attention must be given during the periods when the reactor is shut down, that the electrical power systems continue to fulfill the applicable safety requirements. Also, it must be given when parts of the transmission system near to the NPP are taken out of operation for maintenance or surveillance testing (El-shibini et al., 1995).

The safety systems of the NPP are designed for continuous operation with limited variations in voltage and frequency from the nominal values. In the thermo-hydraulic safety analyses for the NPP, this operating area defines the initial values for pump speed (giving flow and pressure). Hence voltage and frequency of the electricity supply must also be controlled within a defined narrow range (Hassan et al., 2019).

In addition to requiring the grid system and the grid connection to the NPP to be reliable, NPPs also require the grid supply to have sufficient capacity, and to be of an appropriate quality, with both voltage and frequency to be maintained within defined ranges. It may be a requirement of the nuclear regulatory body in the country that the NPP disconnects or shuts down if the grid frequency goes outside the acceptable range, or if the grid voltage becomes so high or low that voltages within the plant are unacceptable.

Briefly, any unexpected sudden disconnect of the NPP from a stable electric grid could trigger a severe imbalance between power generation and feeding causing a sudden drop in grid frequency and voltage. This could even flow into the failure of the grid if added power sources are not joined to the network in time (Kotb et al., 2010).

The main objective of this paper is improving the network performance in all situations of operation (start up, shut down, and maintenance). This improvement in the voltage profile and reducing total power losses of the system will be achieved by using the capacitor devices at optimal allocation (Pyng et al., 2004; Das, 2008).

To solve this optimization problem Genetic Algorithm (GA) technique is used. GA method is a commanding optimization technique analogous to the natural genetic process in natural science. In this paper, the objective function should be changed and the constraint is considered. The new objective function reduces the loss of a grid by considering optimum capacitor placement.

\section{Problem formulation}

The objective of the proposed algorithm aims to minimize the total cost due to capacitor placement, and power loses with constraints that include limits of voltage and size of installed capacitors. The fitness function (FF) is given by (Adail et al., 2018):

$$
\mathrm{FF}=\mathrm{KKp} * \operatorname{real}(\mathrm{SLT})+\operatorname{sum}(\text { cap-cost })+\operatorname{sum}(\mathrm{MM})+
$$
$\operatorname{sum}(\mathrm{CC})$

where: -

- Cap-cost is the capacitor cost price i.e. cap cost (b) $=\mathrm{VVC}^{*} \mathrm{c}(\mathrm{b}), \mathrm{VVC}$ is the cost of KVAR.

- CC is Capacitor Constrain Penalty.

- MM is the Voltage Constrain Penalty typically the minimum and maximum voltage limits.

$V_{\min } \leq V_{i} \leq V_{\max }$

$\mathrm{KKp}$ is the cost of real power loss $=168 \$ /(\mathrm{kW}-$ year) (Mekhamer et al., 2002)

- SLT is the total power loss. 
- Power flow computes from the following equation:

$$
\begin{aligned}
& P_{i+1}=P_{i}-P_{L_{i+1}}-R_{i, i+1} \cdot \frac{\left(P_{i}^{2}+Q_{i}^{2}\right)}{\left|v_{i}^{2}\right|} \\
& Q_{i+1}=Q_{i}-Q_{L_{i+1}}-X_{i, i+1} \cdot \frac{\left(P_{i}^{2}+Q_{i}^{2}\right)}{\left|V_{i}^{2}\right|} \\
& \left|V_{i+1}\right|^{2}=\left|V_{i}\right|^{2}-2\left(P_{i} \cdot R_{i, i+1}+Q_{i} \cdot X_{i, i+1}\right) \\
& +\left(R_{i, i+1}{ }^{2}+X_{i, i+1}{ }^{2}\right) \cdot \frac{\left(P_{i}^{2}+Q_{i}^{2}\right)}{\left|V_{i}^{2}\right|}
\end{aligned}
$$

where $P i$ and $Q i$ are the real and reactive power flowing out of bus i, and $P L i$ and $Q L i$ are the real and reactive load powers at bus i. $R i, i+1$ and $X i, i+1$ are the resistance and reactance of the line section between buses $i$ and $i+1$, respectively. The power loss of the line section connecting buses $i$ and $i+1$ computed as equation

$P_{\text {loss }}(i, i+1)=R_{i, i+1} \cdot \frac{\left(P_{i}^{2}+Q_{i}^{2}\right)}{\left|V^{2}\right|}$

The total power loss of the feeder is calculated by summing up the losses of all line sections of the feeder, The total power loss equation defined by

$$
P_{T, \text { LOSS }}=\sum_{i=0}^{n-1} P_{\text {loss }}(i, i+1)
$$

The bus reactive compensation power equation is

$Q_{i}^{c} \leq \sum_{i=1}^{n} Q_{L_{i}}$

Where $Q_{i}^{c}$ the reactive power is compensated at busi.

\section{Proposed Computational Algorithm}

Now days, there are many of nonlinear techniques using to solve the hard optimization problems. Among the methods which can give us a global optimal solution is GA.

Genetic algorithms use the principle of natural evolution and population genetics to search and arrive near a high-quality global solution. It is essentially built in the principle of natural selection, the procedure that drives biological evolution. GA consists of a population of binary string, searching many peaks in parallel. Following are important terminol- ogy in connection with the genetic algorithm [9]:

The flow chart of our manner is shown in Fig. 1. Genetic Algorithm sets in the core of it and load flow algorithm is used in everywhere that evaluation process is essential.

Finally, base case network is evaluated and its indexes are registered. In the main path of it, Genetic Algorithm meaning full search to find the optimum place, and size for installing capacitors. This routine is programmed under MATLAB software.

\section{Case Study}

After the implementation of the algorithm discussed in previous section, The proposed method has been tested on 16-bus radial distribution system which is presented in table 1 . The program is written using mat lab and tested on windows-7, and the flow chart of the program is given in figure (2). The genetic parameters are population size equal 80 , number of generation equal 100 , mutation probability equal 0.02 , and crossover probability equal 0.08 .

The results are used to compare the network after and before using the proposed technique. The algorithm has been implemented by using MATLAB program.

The technique is applied by choosing the location and size capacitors to minimize the losses and make the voltage within the limits. Fig. 3 is a plot between algorithm of objective function and generation for stander IEEE16-bus system. The new configuration is shown in Fig. 4 and the capacitors value in P.U. is shown in Fig.5.

The optimal values of power losses and Fitness function are 0.0036 , and $4.0199 \mathrm{e}+005$ p.u. and respectively, and the voltage is within the accepted limit. The voltage value in P.U. at all buses after optimization using the present developed technique is shown in Fig.6. 


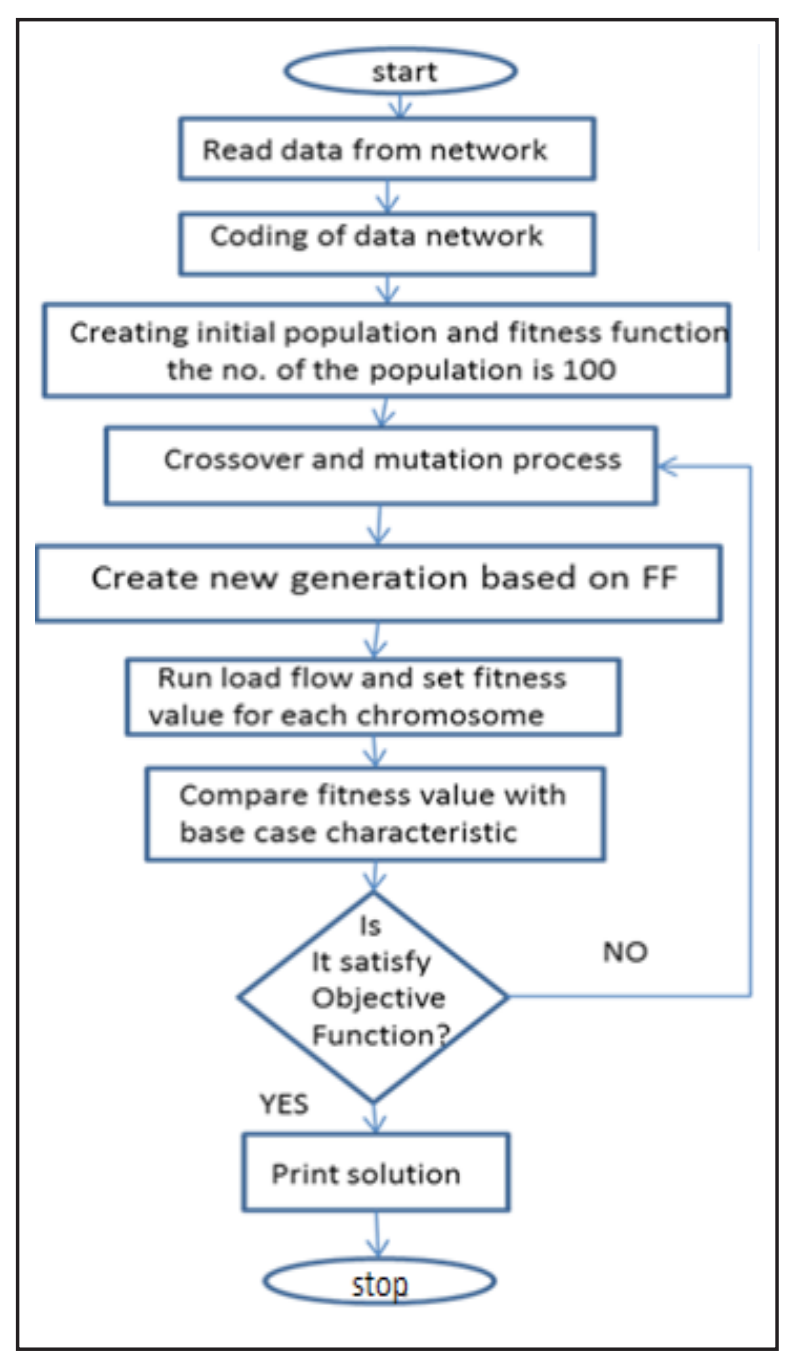

Fig. (1): flow chart of implemented methodology.

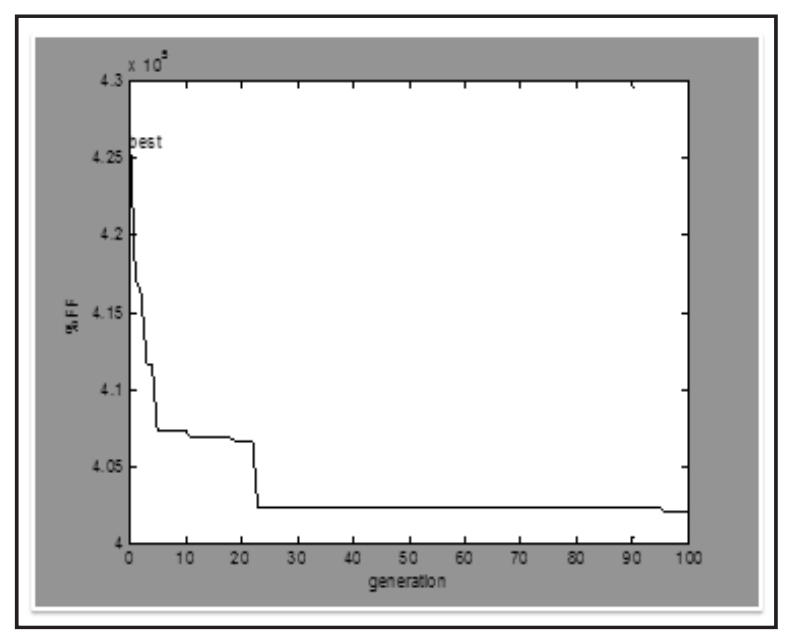

Fig. (3): Objective function for 16- bus system.

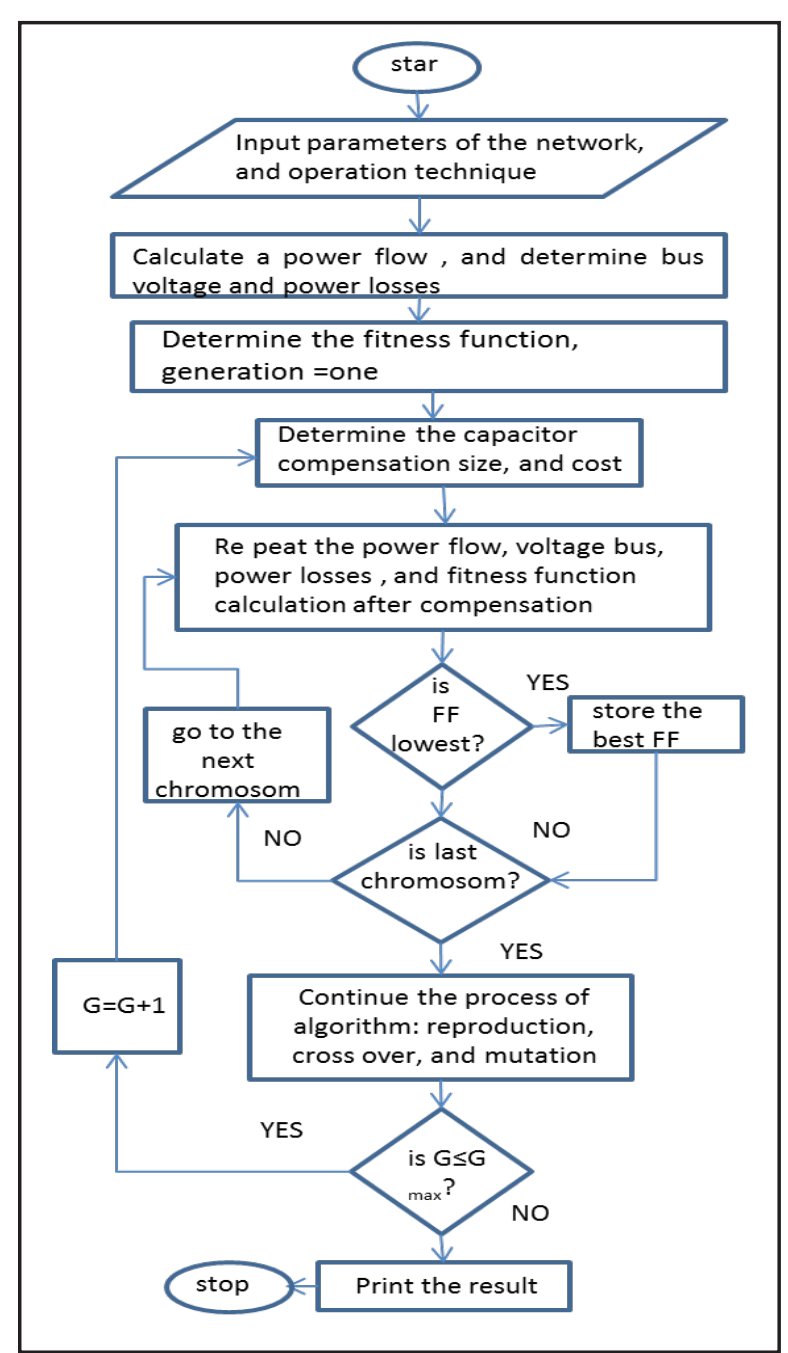

Fig. (2): procedure of the proposed method.

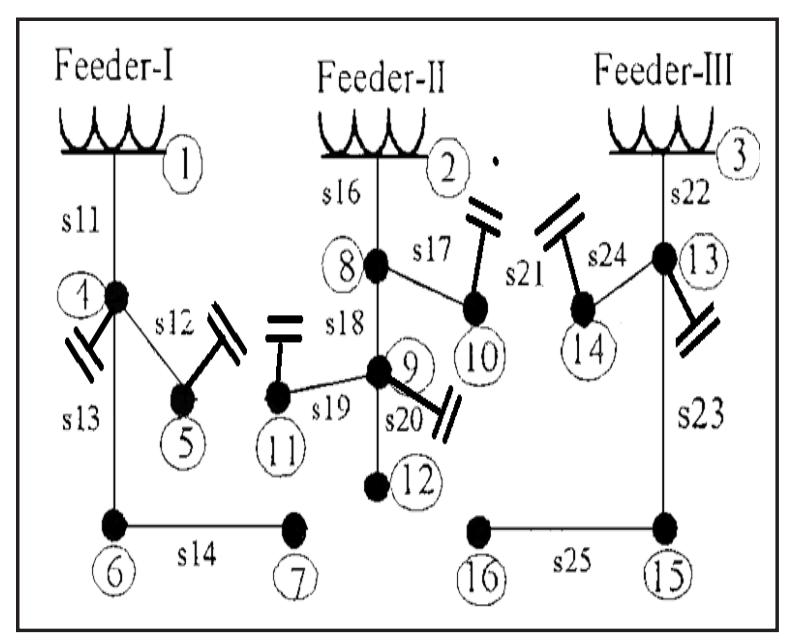

Fig. (4): 16-bus distribution system with capacitors. 
Table (1): Data of the three-feeder simple system.

Base: 100 MVA, $23 \mathrm{kV}$

\begin{tabular}{|c|c|c|c|c|c|}
\hline \multirow{2}{*}{ Bus line } & \multirow{2}{*}{$\begin{array}{c}\text { No. bus } \\
\text { Or sw. }\end{array}$} & $\begin{array}{c}|c| \\
\text { Reactance } \\
(\boldsymbol{p} . \text { u. })\end{array}$ & $\begin{array}{c}\text { Resistance } \\
(\boldsymbol{p} . \boldsymbol{u} \text {. })\end{array}$ & $\begin{array}{c}\boldsymbol{Q} \\
(\boldsymbol{M V A R})\end{array}$ & $\begin{array}{c}\boldsymbol{P} \\
(\boldsymbol{M W})\end{array}$ \\
\cline { 3 - 6 } & Sw1 & 0.10 & 0.075 & 1.6 & 2.0 \\
\hline $\mathbf{1 - 4}$ & Sw2 & 0.11 & .080 & 1.5 & 3.0 \\
\hline $\mathbf{4 - 5}$ & Sw3 & 0.18 & 0.090 & 0.8 & 2.0 \\
\hline $\mathbf{4 - 6}$ & Sw4 & 0.04 & 0.040 & 1.2 & 1.5 \\
\hline $\mathbf{6 - 7}$ & Sw5 & 0.11 & 0.110 & 2.7 & 4.0 \\
\hline $\mathbf{2 - 8}$ & Sw6 & 0.11 & 0.080 & 3.0 & 5.0 \\
\hline $\mathbf{8 - 9}$ & Sw7 & 0.11 & 0.110 & 0.9 & 1.0 \\
\hline $\mathbf{8 - 1 0}$ & Sw8 & 0.11 & 0.110 & 0.1 & 0.6 \\
\hline $\mathbf{9 - 1 1}$ & Sw9 & 0.11 & 0.080 & 2.0 & 4.5 \\
\hline $\mathbf{9 - 1 2}$ & Sw10 & 0.11 & 0.110 & 0.9 & 1.0 \\
\hline $\mathbf{3 - 1 3}$ & Sw11 & 0.12 & 0.090 & 0.7 & 1.0 \\
\hline $\mathbf{1 3 - 1 4}$ & Sw12 & 0.11 & 0.080 & 0.9 & 1.0 \\
\hline $\mathbf{1 3 - 1 5}$ & Sw13 & 0.04 & 0.040 & 1.0 & 2.1 \\
\hline $\mathbf{1 5 - 1 6}$ & Sw14 & 0.04 & 0.040 & - & - \\
\hline $\mathbf{5 - 1 1}$ & Sw15 & 0.04 & 0.040 & - & - \\
\hline $\mathbf{1 0 - 1 4}$ & Sw16 & 0.12 & 0.090 & - & - \\
\hline $\mathbf{7 - 1 6}$ & & & & & \\
\hline
\end{tabular}

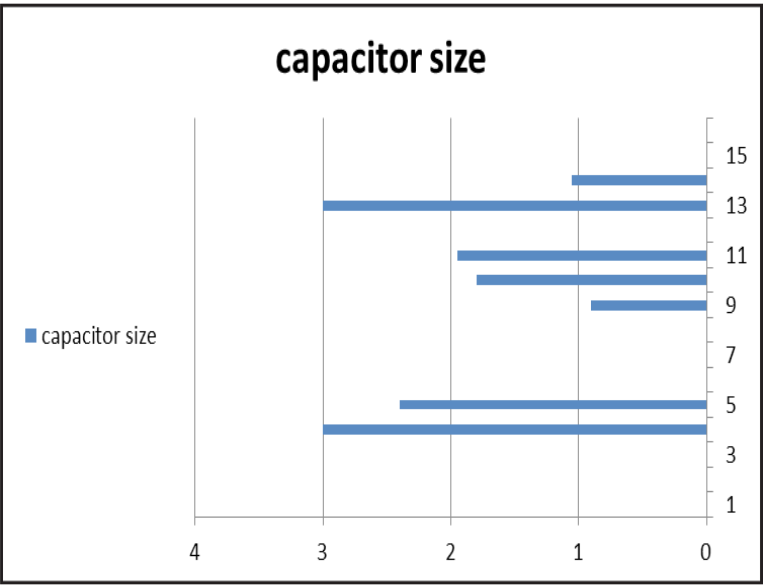

Fig. (5): capacitor size and allocation of 16-bus network.

A comparison between the obtained solutions by the proposed technique at Case study and the Base network configuration is given in Fig.6,7. It is clear that the solution obtained here gives lower losses than the base and the voltage node becomes within the limit.

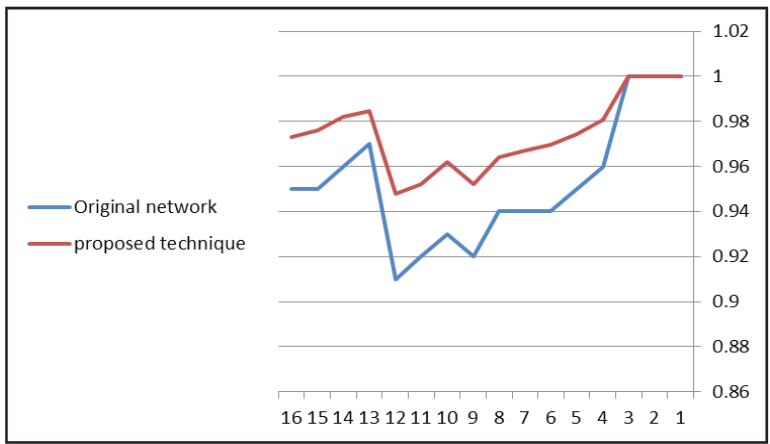

Fig. (6): voltage profile at all buses network.

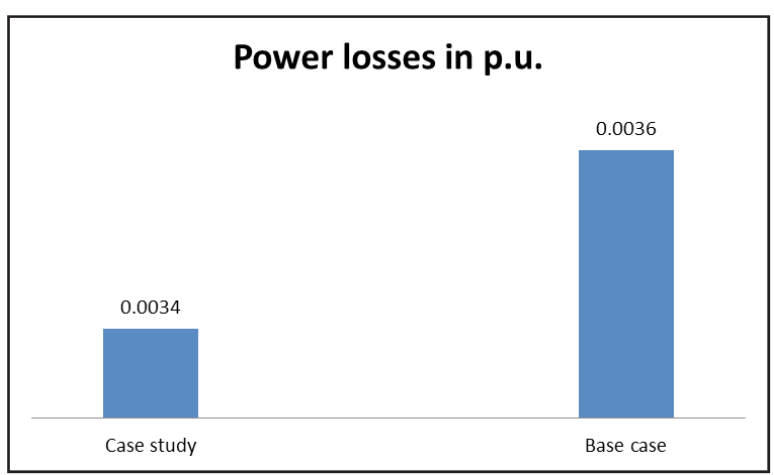

Fig. (7): Compare for power losses between Bus network and Case study. 


\section{CONCLUSION}

The work has been carried out to find the optimal locations and sizes (kVAr) of capacitors placed in radial distribution system to maintain buses voltages with the preset limit, which then reduces the impact effect of the grid network upon NPP. The work also shows that the optimal capacitor placement controlled the flow of reactive power in grid lines and consequently led to decrease lines power losses. Applying the proposed technique on the power grid connected to NPP power house increase network reliability and then reduces the impacts on NPP shutdown and startup. The power distribution Utilities can prioritize their network improvements optimally using the above results. In additional the environmental benefits of energy loss reduction were briefly presented.

\section{REFERENCES}

- Adail, A.S.; Hassan, H.A. and Shaat, M.K (2018): Safety and Reliability of the Electrical System Based on Optimal Facts Allocation for a Research Reactor, Progress in Nuclear Energy, 104.

- Das, D. (2008): Optimal Placement of Capacitors in Radial Distribution System using a Fuzzy-GA method. Int. J. Elec. Power, 30(6-7): 361-367.

- El-shibini, M.; El-marsafawy, M. and Essawy, M. (1995): Improving the Availability of Emergency Power Supply in Nuclear Power Plant. JKSUES, 7: 3-15.

- Kotb, A.S. (2010): Impact the Nuclear Power Plant on Electrical Grid, Phd Thesis, Faculty of Engineering, Al Azhar University. https://inis.iaea.org/search/ search.aspx?orig_q=RN:42009000

- $\quad$ Pyng Chung, J.; Chang, C.FU. and TzongSu C. (2004): Ant Direction Hybrid Differential Evolution for Solving Large Capacitor Placement Problems. IEEE T. POWER SYST., 19(4): 1794 - 1800.
- Hassan, H.A.; Agwa, A. (2019): Onsite Power System Risk Assessment for Nuclear Power Plants Considering Components Ageing, Progress in Nuclear Energy, 110.

- Mekhamer, S.M.; El-Hawary, M.; Mansour, S., Moustafa, M. and Soliman, S.A. (2002): Fuzzy and Heuristic Techniques for Reactive Power Compensation of Radial Distribution Feeders: A Comparative study. Proceedings of IEEE Electrical and Computer Engineering (CCECE), Canadian Conference; DOI: 10.1109/CCECE.2002.1015184. 